PROCEEDINGS OF THE AMERICAN MATHEMATICAL SOCIETY

Volume 124, Number 7, July 1996

\title{
BANACH SPACES IN WHICH EVERY $p$-WEAKLY SUMMABLE SEQUENCE LIES IN THE RANGE OF A VECTOR MEASURE
}

\author{
C. PIÑEIRO
}

(Communicated by Palle E. T. Jorgensen)

\begin{abstract}
Let $X$ be a Banach space. For $1<p<+\infty$ we prove that the identity map $I_{X}$ is $(1,1, p)$-summing if and only if the operator $x^{*} \in X^{*} \rightarrow$ $\sum\left\langle x_{n}, x^{*}\right\rangle e_{n} \in l_{q}$ is nuclear for every unconditionally summable sequence $\left(x_{n}\right)$ in $X$, where $q$ is the conjugate number for $p$. Using this result we find a characterization of Banach spaces $X$ in which every $p$-weakly summable sequence lies inside the range of an $X^{* *}$-valued measure (equivalently, every $p$-weakly summable sequence $\left(x_{n}\right)$ in $X$, satisfying that the operator $\left(\alpha_{n}\right) \in$ $l_{q} \rightarrow \sum \alpha_{n} x_{n} \in X$ is compact, lies in the range of an $X$-valued measure) with bounded variation. They are those Banach spaces such that the identity operator $I_{X} *$ is $(1,1, p)$-summing.
\end{abstract}

Let $X$ be a Banach space. In $[\mathrm{AD}]$ it is proved that every sequence $\left(x_{n}\right)$ in $X$ satisfying $\sum_{n}\left|\left\langle x_{n}, x^{*}\right\rangle\right|^{2}<+\infty$ for all $x^{*} \in X^{*}$ lies inside the range of an $X$-valued measure. Nevertheless, they show a sequence which does not lie in the range of an $X$-valued measure with bounded variation. In $[\mathrm{PR}]$ the authors proved that $X$ is finite dimensional if and only if every nul sequence (equivalently, everyt compact set) in $X$ lies inside the range of an $X$-valued measure having bounded variation. The purpose of this paper is to characterize, given a real number $p \in(1,+\infty)$, the Banach spaces in which every $p$-weakly summable sequence lies inside the range of an $X^{* *}$-valued measure with bounded variation. We start by explaining some basic notation used in this paper. In general, our operator and vector measure terminology and notation follow [Ps] and [DU]. We only consider real Banach spaces. If $X$ is a such space, $B_{X}$ will denote its closed unit ball. The phrase "range of an $X$-valued measure" always means a set of the form $\operatorname{rg}(F)=\{F(A): A \in \Sigma\}$, where $\Sigma$ is a $\sigma$-algebra of subsets of a set $\Omega$ and $F: \Sigma \rightarrow X$ is countably additive. Given $p \geq 1, l_{w}^{p}(X)$ will denote the vector space of all sequences $\left(x_{n}\right)$ in $X$ such that $\sum_{n=1}^{\infty}\left|\left\langle x_{n}, x^{*}\right\rangle\right|^{p}<+\infty$ for all $x^{*} \in X^{*}$. It is easy to see that if $\left(x_{n}\right) \in l_{w}^{p}(X)$, then

$$
\varepsilon_{p}\left(\left(x_{n}\right)\right)=\sup \left\{\left(\sum_{n=1}^{\infty}\left|\left\langle x_{n}, x^{*}\right\rangle\right|^{p}\right)^{1 / p}: x^{*} \in B_{X^{*}}\right\}<+\infty
$$

and $\left(l_{w}^{p}(X), \varepsilon_{p}\right)$ is itself a Banach space.

Received by the editors September 12, 1994 and, in revised form, December 2, 1994.

1991 Mathematics Subject Classification. Primary 46G10; Secondary 47B10.

This research has been partially supported by the D.G.I.C.Y.T., PB 90-893.

(C)1996 American Mathematical Society 
If $\hat{x}=\left(x_{n}\right) \in l_{w}^{p}(X)$ and $P$ is a finite subset of $\mathbb{N}, \hat{x}(P)=\left(x_{n}(P)\right)$ is the sequence defined by

$$
x_{n}(P)= \begin{cases}x_{n} & \text { if } n \in P \\ 0 & \text { if } n \notin P\end{cases}
$$

for all $n \in \mathbb{N}$. $l_{u}^{p}(X)$ will denote the subspace of $l_{w}^{p}(X)$ consisting of the sequences $\hat{x}=\left(x_{n}\right)$ such that the net $(\hat{x}(P))_{P \in \mathcal{F}(\mathbb{N})}$ converges to $\left(x_{n}\right)$ in $l_{w}^{p}(X)$, where $\mathcal{F}(\mathbb{N})$ is the set of all finite subsets of $\mathbb{N}$. Recall that $l_{u}^{1}(X)$ is formed by the unconditionally summable sequences in $X$. We need the following propositions that list some privileges that membership in $l_{w}^{p}(X)$ or in $l_{u}^{p}(X)$ entail.

Proposition A. Let $p>1$ and $X$ be a Banach space. The following statements are equivalent:

(i) $\left(x_{n}\right) \in l_{w}^{p}(X)$. $l_{q}$.

(ii) The series $\sum_{n=1}^{\infty} \alpha_{n} x_{n}$ converges unconditionally for every sequence $\left(\alpha_{n}\right) \in$

(iii) The map $\left(\alpha_{n}\right) \in l_{q} \rightarrow \sum_{n=1}^{\infty} \alpha_{n} x_{n} \in X$ defines a bounded operator.

Proposition B. Let $p \geq 1$. If $\left(x_{n}\right) \in l_{u}^{p}(X)$, then the operator $\left(\alpha_{n}\right) \in l_{q} \rightarrow$ $\sum_{n=1}^{\infty} \alpha_{n} x_{n} \in X$ is compact.

\section{Main Result}

Throughout this section $X$ will be a Banach space and $p \in(1,+\infty)$.

Theorem 1. The following statements are equivalent:

(i) For every unconditionally sequence $\left(x_{n}\right)$ in $X$ the operator $x^{*} \in X^{*} \rightarrow$ $\sum_{n=1}^{\infty}\left\langle x_{n}, x^{*}\right\rangle e_{n} \in l_{q}$ is nuclear.

(ii) There exists a constant $c>0$ such that

$$
\begin{aligned}
&\left|\sum_{k=1}^{n}\left\langle x_{k}, x_{k}^{*}\right\rangle\right| \leq c \sup \left\{\sum_{k=1}^{n}\left|\left\langle x_{k}, x^{*}\right\rangle\right|:\left\|x^{*}\right\| \leq 1\right\} \\
& \cdot \sup \left\{\left(\sum_{k=1}^{n}\left|\left\langle x, x_{k}^{*}\right\rangle\right|^{p}\right)^{1 / p}:\|x\| \leq 1\right\}
\end{aligned}
$$

for all $\left\{x_{1}, \ldots, x_{n}\right\} \subset X$ and $\left\{x_{1}^{*}, \ldots, x_{n}^{*}\right\} \subset X^{*}$.

Proof. (i) $\Rightarrow$ (ii) We consider the linear map

$$
\hat{x}=\left(x_{n}\right) \in l_{u}^{1}(X) \rightarrow T_{\hat{x}} \in \mathcal{N}\left(X^{*}, l_{q}\right)
$$

defined by $T_{\hat{x}}\left(x^{*}\right)=\sum\left\langle x_{n}, x^{*}\right\rangle e_{n}$ for all $x^{*} \in X^{*}\left(\left\{e_{n}: n \in \mathbb{N}\right\}\right.$ is the unit basis of $l_{q}$ ). It has closed graph, so there exists a positive constant $c$ so that

$$
\nu\left(\sum_{n=1}^{\infty} x_{n} \otimes e_{n}: X^{*} \rightarrow l_{q}\right) \leq c \sup \left\{\sum_{n=1}^{\infty}\left|\left\langle x_{n}, x^{*}\right\rangle\right|:\left\|x^{*}\right\| \leq 1\right\}
$$

for all $\left(x_{n}\right) \in l_{u}^{1}(X)$. By a standard argument we obtain

$$
\nu\left(\sum_{n=1}^{m} x_{n} \otimes e_{n}: X^{*} \rightarrow l_{q}^{m}\right) \leq c \sup \left\{\sum_{n=1}^{m}\left|\left\langle x_{n}, x^{*}\right\rangle\right|:\left\|x^{*}\right\| \leq 1\right\}
$$

for all $m \in \mathbb{N}$ and $\left\{x_{1}, \ldots, x_{m}\right\} \subset X$. 
Now, given $\left\{x_{1}, \ldots, x_{m}\right\} \subset X$ and $\left\{x_{1}^{*}, \ldots, x_{m}^{*}\right\} \subset X^{*}$, define two operators $v: l_{q}^{m} \rightarrow X^{*}$ and $u: X^{*} \rightarrow l_{q}^{m}$ by

$$
v\left(\alpha_{i}\right)=\sum_{i=1}^{m} \alpha_{i} x_{i}^{*} \quad \text { and } \quad u\left(x^{*}\right)=\sum_{i=1}^{m}\left\langle x_{i}, x^{*}\right\rangle e_{i}
$$

Note that $\operatorname{tr}(u \circ v)=\sum_{i=1}^{m}\left\langle x_{i}, x_{i}^{*}\right\rangle$, so we have

$$
\begin{aligned}
\left|\sum_{i=1}^{m}\left\langle x_{i}, x_{i}^{*}\right\rangle\right| & \leq \nu(u \circ v) \leq \nu(u)\|v\| \\
& =\nu(u) \sup \left\{\left(\sum_{i=1}^{m}\left|\left\langle x, x_{i}^{*}\right\rangle\right|^{p}\right)^{1 / p}:\|x\| \leq 1\right\}
\end{aligned}
$$

and using (2) we obtain

$$
\begin{aligned}
&\left|\sum_{k=1}^{n}\left\langle x_{k}, x_{k}^{*}\right\rangle\right| \leq c \sup \left\{\sum_{k=1}^{n}\left|\left\langle x_{k}, x^{*}\right\rangle\right|:\left\|x^{*}\right\| \leq 1\right\} \\
& \cdot \sup \left\{\left(\sum_{k=1}^{n}\left|\left\langle x, x_{k}^{*}\right\rangle\right|^{p}\right)^{1 / p}:\|x\| \leq 1\right\} .
\end{aligned}
$$

(ii) $\Rightarrow$ (i) Given $\left(x_{n}^{*}\right) \in l_{w}^{p}\left(X^{*}\right)$, we define a linear form $\phi$ by

$$
\left(x_{n}\right) \in l_{u}^{1}(X) \rightarrow \sum_{n=1}^{\infty}\left\langle x_{n}, x_{n}^{*}\right\rangle \in \mathbb{R}
$$

By (ii) $\phi \in l_{u}^{1}(X)^{*}$ and $\|\phi\| \leq c\left\|\left(x_{n}^{*}\right)\right\|_{p}$. So, the linear map $x \in X \rightarrow\left(\left\langle x, x_{n}^{*}\right\rangle\right) \in l_{\infty}$ is integral (see [DU, p. 232]). Equivalently, $x \in X \rightarrow\left(\left\langle x, x_{n}^{*}\right\rangle\right) \in c_{0}$ is integral. Then, so is its adjoint $\left(\alpha_{n}\right) \in l_{1} \rightarrow \sum \alpha_{n} x_{n}^{*} \in X^{*}$. Therefore, the linear map $\psi$ defined by

$$
\left(x_{n}^{*}\right) \in l_{w}^{p}\left(X^{*}\right) \rightarrow \sum_{n=1}^{\infty} e_{n} \otimes x_{n}^{*} \in I\left(l_{1}, X^{*}\right)
$$

is well defined and $\|\psi\| \leq c$. Now denote the restriction map of $\psi$ to $l_{u}^{p}\left(X^{*}\right)$ by $\psi_{u}$. Since $\hat{x}^{*}=\lim _{P} \hat{x}^{*}(P)$ for all $\hat{x}^{*} \in l_{u}^{p}\left(X^{*}\right)$, it follows that $\psi_{u}$ takes all its values in $\mathcal{N}\left(l_{1}, X^{*}\right)$ (note that $\mathcal{N}\left(l_{1}, X^{*}\right)$ is a subspace of $I\left(l_{1}, X^{*}\right)$ because $\left(l_{1}\right)^{*}$ has the metric approximation property). If we also denote the operator

$$
\left(x_{n}^{*}\right) \in l_{u}^{p}\left(X^{*}\right) \rightarrow \sum_{n=1}^{\infty} e_{n} \otimes x_{n}^{*} \in \mathcal{N}\left(l_{1}, X^{*}\right)
$$

by $\psi_{u}$, then $\left(\psi_{u}\right)^{*}$ maps $B\left(X^{*}, l_{1}\right)$ into $l_{u}^{p}\left(X^{*}\right)^{*}$. In particular, for all $\left(x_{n}\right) \in l_{u}^{1}(X)$, the operator $x^{*} \in X^{*} \rightarrow \sum\left\langle x_{n}, x^{*}\right\rangle e_{n} \in l_{q}$ is integral. This completes the proof because nuclear and integral operators into a reflexive space are the same. 
Recall that an operator $T: X \rightarrow Y$ is called $(r, q, p)$-summing if there is a constant $c \geq 0$ such that

$$
\begin{aligned}
\left(\sum_{k=1}^{n}\left|\left\langle T x_{k}, y_{k}^{*}\right\rangle\right|^{r}\right)^{1 / r} \leq & c \sup _{x^{*} \in B_{X^{*}}}\left(\sum_{k=1}^{n}\left|\left\langle x_{k}, x^{*}\right\rangle\right|^{q}\right)^{1 / q} \\
& \cdot \sup _{y \in B_{Y}}\left(\sum_{k=1}^{n}\left|\left\langle y, y_{k}^{*}\right\rangle\right|^{p}\right)^{1 / p}
\end{aligned}
$$

for all finite families of elements $x_{1}, \ldots, x_{n} \in X$ and functionals $y_{1}^{*}, \ldots, y_{n}^{*} \in Y^{*}$. So, Theorem 1 gives us a characterization of Banach spaces $X$ for which $I_{X}$ is $(1,1, p)$-summing.

An operator $T: X \rightarrow Y$ is $(p, q)$-summing if there is a constant $c \geq 0$ such that

$$
\left(\sum_{k=1}^{n}\left\|T x_{k}\right\|^{p}\right)^{1 / p} \leq c \sup \left\{\left(\sum_{k=1}^{n}\left|\left\langle x_{k}, x^{*}\right\rangle\right|^{q}\right)^{1 / q}:\left\|x^{*}\right\| \leq 1\right\}
$$

for all finite subset $\left\{x_{1}, \ldots, x_{n}\right\}$ of $X$.

Following [Ps] we will say that a Banach space $X$ satisfies Grothendieck's Theorem (in short, $X$ is a G.T. space) if $B\left(X, l_{2}\right)=\Pi_{1}\left(X, l_{2}\right)$. The next proposition shows the relationship between the Banach spaces $X$ for which $I_{X}$ is absolutely $(1,1, p)$-summing and the above classes.

Proposition 2. (i) If $X$ is a G.T. space, then $I_{X}$ is $(1,1, p)$-summing for $1<p \leq$ 2.

(ii) If $1<p<+\infty$ and $T \in B(X, Y)$, then

$$
T \text { is }(1,1, p) \text {-summing } \Rightarrow T \text { is }(q, 1) \text {-summing. }
$$

Proof. If $\left(x_{n}\right) \in l_{u}^{1}(X)$, then the operator $T: x^{*} \in X^{*} \rightarrow \sum\left\langle x_{n}, x^{*}\right\rangle e_{n} \in l_{q}$ admits the following factorization

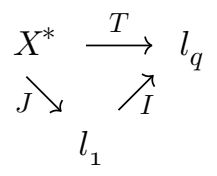

where $I: l_{1} \rightarrow l_{q}$ is the natural inclusion and $J: X^{*} \rightarrow l_{1}$ is defined by $J x^{*}=$ $\left(\left\langle x_{n}, x^{*}\right\rangle\right)$ for all $x^{*} \in X^{*}$. I is obviously 1 -summing and $J$ is 2 -summing by [Ps, 6.6.2], so $T$ is nuclear.

(ii) If $T$ is $(1,1, p)$-summing there is a constant $c \geq 0$ such that

$$
\left|\sum_{i=1}^{n}\left\langle T x_{i}, y_{i}^{*}\right\rangle\right| \leq \operatorname{csup}\left\{\sum_{i=1}^{n}\left|\left\langle x_{i}, x^{*}\right\rangle\right|:\left\|x^{*}\right\| \leq 1\right\} \cdot \varepsilon_{p}\left(\left(y_{i}^{*}\right)_{i=1}^{n}\right)
$$

for all $\left\{x_{1}, \ldots, x_{n}\right\} \subset X$ and $\left\{y_{1}^{*}, \ldots, y_{n}^{*}\right\} \subset Y^{*}$. Given $\left\{x_{1}, \ldots, x_{n}\right\} \subset X$, choose $y_{i}^{*} \in B_{Y^{*}}$ so that $\left|\left\langle T x_{i}, y_{i}^{*}\right\rangle\right|=\left\|T x_{i}\right\|$ for each $i \leq n$. By (3) we have

$$
\begin{aligned}
\sum_{i=1}^{n}\left|\alpha_{i}\right|\left\|T x_{i}\right\| & =\sum_{i=1}^{n}\left|\alpha_{i}\right|\left|\left\langle T x_{i}, y_{i}^{*}\right\rangle\right| \leq c \varepsilon_{1}\left(\left(x_{i}\right)_{i=1}^{n}\right) \varepsilon_{p}\left(\left(\alpha_{i} y_{i}^{*}\right)_{i=1}^{n}\right) \\
& \leq c \varepsilon_{1}\left(\left(x_{i}\right)_{i=1}^{n}\right)\left\|\left(\alpha_{i}\right)\right\|_{p}
\end{aligned}
$$


for all $\left(\alpha_{i}\right) \in l_{p}^{n}$. Then

$$
\left(\sum_{i=1}^{n}\left\|T x_{i}\right\|^{q}\right)^{1 / q} \leq c \sup \left\{\sum_{i=1}^{n}\left|\left\langle x_{i}, x^{*}\right\rangle\right|:\left\|x^{*}\right\| \leq 1\right\}
$$

for all $\left\{x_{1}, \ldots, x_{n}\right\} \subset X$.

In $[\mathrm{P}, 17.1 .6]$, Pietsch formulated the following conjecture: for $1 / r>1 / q+1 / p-$ $1 / 2, I_{X}$ is $(r, q, p)$-summing if and only if $X$ is finite dimensional. The conjecture is true for $q=r=1$. Certainly, let $p>2$. If $I_{X}$ is $(1,1, p)$-summing, it follows from Proposition 2(ii) that $I_{x}$ is $(q, 1)$-summing. By [P, Theorem 17.2.7.] $X$ has to be finite dimensional since $q<2$.

\section{SEquences in the RANGe of A VECTOR MeAsure WITH BOUNDED VARIATION}

In this section we use Theorem 1 to obtain a characterization of Banach spaces $X$ for which every $p$-weakly summable sequence $\left(x_{n}\right)$ in $X$ lies inside the range of an $X^{* *}$-valued measure having bounded variation. The following lemma collects some elementary facts we need (see [Pi 2]).

Lemma 3. Let $X$ be a Banach space. If $\hat{x}=\left(x_{n}\right)$ is a bounded sequence in $X$, we consider the linear operator $T_{\hat{x}}: l_{1} \rightarrow X$ defined by $T_{\hat{x}}\left(\alpha_{n}\right)=\sum \alpha_{n} x_{n}$ for all $\left(\alpha_{n}\right) \in l_{1}$. Then the following assertions hold:

(i) $\left(x_{n}\right)$ lies inside the range of an $X^{* *}$-valued measure with bounded variation iff $T_{\hat{x}}$ is integral.

(ii) $\left(x_{n}\right)$ lies inside the range of an $X$-valued measure with bounded variation iff $T_{\hat{x}}$ is Pietsch-integral.

Now we are ready to face our problem.

Theorem 4. Let $X$ a Banach space and $1<p<+\infty$. The following statements are equivalent:

(i) Every sequence $\left(x_{n}\right) \in l_{w}^{p}(X)$ lies inside the range of an $X^{* *}$-valued measure with bounded variation.

(ii) Every sequence $\left(x_{n}\right) \in l_{w}^{p}(X)$, satisfying that the operator $\left(\alpha_{n}\right) \in l_{q} \rightarrow$ $\sum \alpha_{n} x_{n} \in X$ is compact, lies inside the range of an $X$-valued measure with bounded variation.

(iii) Every sequence $\left(x_{n}\right) \in l_{u}^{p}(X)$ lies inside the range of an $X$-valued measure with bounded variation.

(iv) $I_{X^{*}}$ is $(1,1, p)$-summing.

Proof. (i) $\Rightarrow$ (ii) By Lemma 3, we can consider the linear map

$$
\phi: \hat{x} \in l_{w}^{p}(X) \rightarrow T_{\hat{x}} \in I\left(l_{1}, X\right) .
$$

It is continuous because its graph is closed. Since $\left(l_{q}\right)^{*}$ has the approximation property, for each sequence $\hat{x}=\left(x_{n}\right) \in l_{w}^{p}(X)$ satisfying that the operator $\left(\alpha_{n}\right) \in$ $l_{q} \rightarrow \sum \alpha_{n} x_{n} \in X$ is compact, there exists a sequence $\left(\hat{y}_{k}\right)$ in $l_{w}^{p}(X)$ such that $\hat{x}=\lim _{k \rightarrow+\infty} \hat{y}_{k}$ in $l_{w}^{p}(X)$ and each sequence $\hat{y}_{k}$ is finite dimensional. Then $\phi\left(\hat{y}_{k}\right)$ belongs to $\mathcal{N}\left(l_{1}, X\right)$ for all $k \in \mathbb{N}$. By continuity, so does $\phi(\hat{x})$ (recall that $\mathcal{N}\left(l_{1}, X\right)$ is a closed subspace of $\left.I\left(l_{1}, X\right)\right)$. Hence, we have proved that such a sequence 
$\left(x_{n}\right) \in l_{w}^{p}(X)$ actually lies inside a sum of segments

$$
\sum\left[-z_{n}, z_{n}\right]=\left\{\sum \alpha_{n} z_{n}:\left(\alpha_{n}\right) \in l_{\infty},\left\|\left(\alpha_{n}\right)\right\|_{\infty} \leq 1\right\}
$$

where $\sum\left\|z_{n}\right\|<+\infty$ (see [Pi 1]).

(ii) $\Rightarrow$ (iii) It is obvious because the operator

$$
\left(\alpha_{n}\right) \in l_{q} \rightarrow \sum \alpha_{n} x_{n} \in X
$$

is compact for each sequence $\left(x_{n}\right) \in l_{n}^{p}(X)$.

(iii) $\Rightarrow$ (iv) Now we consider the linear map

$$
\psi: \hat{x} \in l_{u}^{p}(X) \rightarrow T_{\hat{x}} \in I\left(l_{1}, X\right) .
$$

Having a closed graph, $\psi$ is continuous. Since $\hat{x}=\lim _{P} \hat{x}(P)$ for every sequence $\hat{x} \in l_{u}^{p}(X)$, it follows that $\psi$ takes its values into $\mathcal{N}\left(l_{1}, X\right)$. As mentioned earlier, using the trace duality it is easy to prove that $\psi^{*}$ takes every $\left(x_{n}^{*}\right) \in l_{u}^{1}\left(X^{*}\right)$ in $\sum x_{n}^{*} \otimes e_{n} \in I\left(X, l_{q}\right)$. Again the reflexivity of $l_{q}$ yields (iv).

(iv) $\Rightarrow$ (i) In the same way as in the proof of Theorem 1 we can prove that the linear map

$$
\left(x_{n}^{* *}\right) \in l_{w}^{p}\left(X^{* *}\right) \rightarrow \sum_{n=1}^{\infty} e_{n} \otimes x_{n}^{* *} \in I\left(l_{1}, X^{* *}\right)
$$

is well defined and continuous. In particular, it follows from the above lemma that every $\left(x_{n}\right) \in l_{w}^{p}(X)$ lies inside the range of an $X^{* *}$-valued measure of bounded variation.

In view of Theorem 4 and the notes at the end of section 1 , for $p>2$, only finitedimensional Banach spaces $X$ have the property that every sequence $\left(x_{n}\right) \in l_{w}^{p}(X)$ lies inside the range of an $X$-valued measure having bounded variation. That is why from now on we only consider $p \in[1,2]$.

\section{Final notes AND EXAmples}

It is well known that every sequence $\left(x_{n}\right) \in l_{w}^{1}(X)$ lies inside the range of an $X$-valued measure with bounded variation. In fact, the vector measure $F$ defined by

$$
F(A)=2 \sum_{n=1}^{\infty}\left(\int_{A} r_{n}(t) d t\right) x_{n}
$$

for any Lebesgue measurable subset $A$ of $[0,1]$, has bounded variation whenever $\left(x_{n}\right) \in l_{w}^{1}(X)$. In $[\mathrm{AD}]$ it is proved that $\left\{x_{n}: n \in \mathbb{N}\right\} \subset r g(F)$. Then, given an infinite-dimensional Banach space $X$, we can consider the set $r(X)$ formed by all real numbers $r \in[1,2]$ such that every sequence $\left(x_{n}\right) \in l_{w}^{r}(X)$ lies inside the range of an $X$-valued measure having bounded variation. Then $r(X)$ is an interval whose bounds are 1 and $\sup (r(X))$. In the following we will determine the set $r(X)$ for some classical Banach spaces.

(i) $r(X)=[1,2]$ for every Banach space $X$ satisfying:

(a) $X^{*}$ is a G.T. space,

(b) $X$ is a dual space. 
By Proposition 2(i), $I_{X^{*}}$ is $(1,1, r)$-summing for all $r \in(1,2]$. Then Theorem 4 implies that $r \in r(X)$ for all $r \in[1,2]$.

In particular, if $\mu$ is a $\sigma$-finite positive measure, $r\left(L^{\infty}(\mu)\right)=[1,2]$.

(ii) $r\left(l_{p}\right)=\{1\}$ for $1 \leq p<+\infty$.

$I_{p}$ will denote the identity map $l_{p} \rightarrow l_{p}$. First, we consider the case $p=1$. If $\left(e_{n}^{*}\right)$ denotes the unit basis of $l_{\infty}=\left(l_{1}\right)^{*}$, then $\left(e_{n}^{*}\right) \in l_{w}^{1}\left(l_{\infty}\right)$. Since $\sum\left\|e_{n}^{*}\right\|^{s}=\infty$ for $s \geq 1$ it follows that $I_{\infty}$ cannot be $(s, 1)$-summing for $s \geq 1$. So, Proposition 2(ii) tells us that $I_{\infty}$ is not $(1,1, r)$-summing for $r>1$. By Theorem $4, r\left(l_{1}\right)=\{1\}$.

Now suppose $1<p<+\infty$.

Claim. $r\left(l_{p}\right) \cap(1, q)=\emptyset$. Let $r \in r\left(l_{p}\right) \cap(1, q)$. Theorems 1 and 4 assure us that there is a constant $c \geq 0$ such that

$$
\sum_{i=1}^{n}\left|\left\langle x_{i}, x_{i}^{*}\right\rangle\right| \leq c \varepsilon_{1}\left(\left(x_{i}^{*}\right)_{i=1}^{n}\right) \varepsilon_{r}\left(\left(x_{i}\right)_{i=1}^{n}\right)
$$

for all $\left\{x_{1}, \ldots, x_{n}\right\} \subset l_{p}$ and $\left\{x_{1}^{*}, \ldots, x_{n}^{*}\right\} \subset l_{q}$. Given $\left(\alpha_{n}\right) \in l_{q}$ and $\left(\beta_{n}\right) \in l_{u}$ with $u=r q(q-r)^{-1}$, define $x_{n}^{*}=\alpha_{n} e_{n}^{*}$ and $x_{n}=\beta_{n} e_{n}$ for all $n \in \mathbb{N}$. From (4) we get

$$
\sum_{i=1}^{m}\left|\alpha_{i}\right|\left|\beta_{i}\right| \leq c \varepsilon_{1}\left(\left(\alpha_{i} e_{i}^{*}\right)_{i=1}^{m}\right) \varepsilon_{r}\left(\left(\beta_{i} e_{i}\right)_{i=1}^{m}\right)
$$

for all $m \in \mathbb{N}$. Applying Holder's inequality we obtain

$$
\varepsilon_{1}\left(\left(\alpha_{i} e_{i}^{*}\right)_{i=1}^{m}\right) \leq\left\|\left(\alpha_{n}\right)\right\|_{q} \varepsilon_{p}\left(\left(e_{n}^{*}\right)\right)=\left\|\left(\alpha_{n}\right)\right\|_{q}
$$

and

$$
\varepsilon_{r}\left(\left(\beta_{i} e_{i}\right)_{i=1}^{m}\right) \leq \varepsilon_{q}\left(\left(e_{n}\right)\right)\left\|\left(\beta_{n}\right)\right\|_{u}=\left\|\left(\beta_{n}\right)\right\|_{u} .
$$

Then, for all $m \in \mathbb{N}$ and $\left(\alpha_{n}\right) \in l_{q}$, we have

$$
\sum_{i=1}^{m}\left|\alpha_{i}\right|\left|\beta_{i}\right| \leq c\left\|\left(\alpha_{n}\right)\right\|_{q}\left\|\left(\beta_{n}\right)\right\|_{u} .
$$

This implies that $\left(\beta_{n}\right) \in l_{p}=\left(l_{q}\right)^{*}$. Choosing $\left(\beta_{n}\right) \in l_{u} \backslash l_{p}$ we fall in a contradiction since $r q(q-r)^{-1}>p$.

With our claim established we already have proved that $r\left(l_{p}\right)=\{1\}$ for $p<2$. Finally, we are going to show that $r\left(l_{p}\right) \cap[q, 2]=\emptyset$ for $p \geq 2$. This is the easy part. Certainly, the identity map $l_{1} \rightarrow l_{p}$ is not nuclear, hence Lemma 3(ii) allows us to conclude that the sequence $\left(e_{n}\right)$ does not lie inside the range of an $l_{p}$-valued measure of bounded variation. Nevertheless, $\left(e_{n}\right) \in l_{w}^{r}\left(l_{p}\right)$ for all $r \geq q$. Thus $[q, 2] \cap r\left(l_{p}\right)=\emptyset$.

(iii) $r(X)=\{1\}$ for all infinite-dimensional $\mathcal{L}_{p}$-space $X$ with $1 \leq p<+\infty$.

By [LP, Proposition 7.3], $X$ has a complemented subspace $H$ isomorphic to $l_{p}$. Then $r(X) \subset r(H)=r\left(l_{p}\right)=\{1\}$.

\section{REFERENCES}

[AD] R. Anantharaman and J. Diestel, Sequences in the range of a vector measure, Anna. Soc. Math. Polon. Ser. I Comment. Math. Prace Mat. 30 (1991), 221-235. MR 92g:46049

[DU] J. Diestel and J. J. Uhl, Vector measures, Math. Surveys Monographs, vol. 15, Amer. Math. Soc., Providence, RI, 1977. MR 56:12216

[LP] J. Lindenstrauss and Pelczynski, Absolutely summing operators in $\mathcal{L}_{p}$-spaces and their applications, Studia Math. 29 (1968), 275-326. MR 37:6743

[P] A. Pietsch, Operator ideals, North-Holland, Amsterdam, 1980. MR 81j:47001 
[Pi 1] C. Piñeiro, Operators on Banach spaces taking compact sets inside ranges of vector measures, Proc. Amer. Math. Soc. 116 (1992), 1031-1040. MR 93b:47076

[Pi 2] _ Sequences in the range of a vector measure with bounded variations, Proc. Amer. Math. Soc. 123 (1995), 3329-3334. CMP 95:16

[PR] C. Piñeiro and L. Rodriguez-Piazza, Banach spaces in which every compact lies inside the range of a vector measure, Proc. Amer. Math. Soc. 114 (1992), 505-517. MR 92e:46038

[Ps] G. Pisier, Factorization of linear operators and geometry of Banach spaces, CBMS Regional Conf. Ser. in Math., vol. 60, Amer. Math. Soc., Providence, RI, 1986. MR 88a:47020

[T] N. Tomczak-Jaegermann, Banach-Mazur distances and finite-dimensional operator ideals, Pitman Monographs Surveys Pure Appl. Math., vol. 38, Longman Sci. Tech., Harlow, 1989. MR 90k:46039

Departamento de Análisis Matemático, Facultad de Matemáticas, Universidad de Sevilla, Aptdo. 1160, Sevilla, 41080, Spain

Current address: Departamento de Matemáticas, Escuela Politécnica Superior, Universidad de Huelva, 21810 La Rábida, Huelva, Spain 